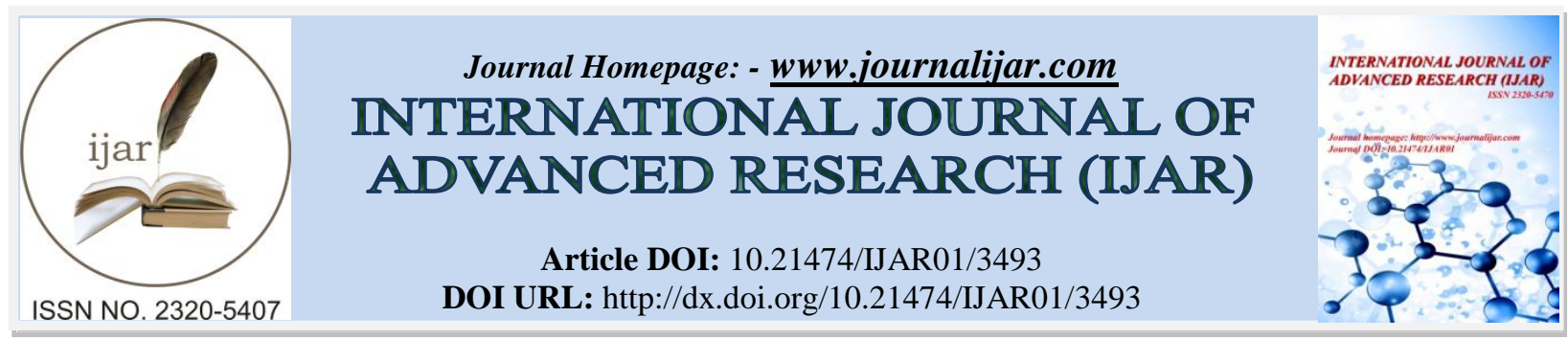

RESEARCH ARTICLE

\title{
FREQUENCY OF PERIPHERAL NEUROPATHY IN PATIENTS WITH CHRONIC KIDNEY DISEASE: A CLINICAL STUDY.
}

\author{
Dr. Muhammad Mujtaba Hashir ${ }^{1}$, Zainab bahrani ${ }^{2}$, Fatimah Khater ${ }^{2}$ and Zainab Alwan ${ }^{2}$. \\ 1. Assistant Professor, Department of Medicine, King Faisal University, Al-Ahsa, Saudi Arabia. \\ 2. King Faisal University, Al-Ahsa, Saudi Arabia.
}

\section{Manuscript Info}

Manuscript History

Received: 13 January 2017

Final Accepted: 10 February 2017

Published: March 2017

Key words:-

CKD, Diabetes, ESRD, Hemodialysis, Hypertension, Peripheral Neuropathy.

\begin{abstract}
Chronic kidney disease (CKD) is one of the major health problems in modern world. Peripheral neuropathy is considered to be an important complication in these patients. It can cause foot ulcers and may lead to circulatory problems. Regular and timely screening has the potential in reducing the incidence of complications due to peripheral neuropathy. The present study is conducted to measure the frequency of peripheral neuropathy among patients with CKD being treated with hemodialysis.

Objectives:-To measure the frequency of peripheral neuropathy among patients with chronic kidney disease.

Methods:- The current study was conducted in Al-Jabar Kidney Center under the Ministry of Health in Al-Ahsa region, Eastern province, Kingdom of Saudi Arabia. Peripheral neuropathy was evaluated clinically in patients included.

Results:- About $62.5 \%$ patients of CKD were found to have peripheral neuropathy clinically. Out of these $78 \%$ were diabetics while $94 \%$ were hypertensive.
\end{abstract}

Copy Right, IJAR, 2017,. All rights reserved.

\section{Introduction:-}

Chronic kidney disease (CKD) is a common disease that is characterized by pathological kidney abnormalities with persistent proteinuria. CKD is divided into 5 stages, based on glomerular filtration rate (GFR). In stage 1 and stage 2, GFR is near normal with functional and structural defects (e.g, hematuria and persistent proteinuria). Stage 3 has low risk of progression to kidney failure, the GFR being $30-59 \mathrm{~mL} / \mathrm{min}$. While in late stages, like 4 and 5 , there is loss of significant renal function. The last stage (stage 5), called renal failure or end stage renal disease (ESRD), patients need dialysis or kidney transplant. End stage renal disease (ESRD) is the last stage of chronic kidney disease. It is irreversible condition of impairment of renal function when the kidney is unable to detect the balance of fluid and electrolytes, and unable to excrete waste product so, the patient develops uremia. The ESRD has more prevalence among diabetic and hypertensive patients, and is associated with various complications; like cardiac abnormality, neuropathy, hyperlipidemia, anemia and metabolic bone disease. Neurological complications are common in patient with ESRD, secondary to uremic state, affecting nervous system at any level; central and peripheral. So, patient may suffer from prolonged disability, weakness, loss of sensations, and alteration of mental state. Uremic peripheral neuropathy is symmetrical distal polyneuropathy with slowly progressive sensorimotor axonal neuropathy. The presentation of uremic peripheral neuropathy varies from patient to patient. Patients may present by sensory manifestations insidiously, which may consist of tingling and prickling sensation in the lower 
extremities, paresthesias, loss of touch, pain and temperature sensations. The rationale of this study is, if there is high frequency of involvement of peripheral neuropathy (loss of position, vibration, pain and touch) in ESRD patients on frequent hemodialysis; then screening for this should be among the routine clinical examination of patients with CKD.

\section{Material and methods:-}

The study aims to first determine the frequency of peripheral neuropathy in hemodialysis patient and plan for treatment and prevention if found. The setting was in hemodialysis department, the duration of the study was: 5 months. We used Cross-sectional Study design. Sample size of 80, of which 42 were females and 38 were males, the age group was from 30 to 60 years. Purposive non-probability sample technique. We selected all patients of 30 to 60 years of age, who had ESRD and were on hemodialysis. They varied from medical illness free to patient with hypertension or diabetes. We excluded patients who were previously diagnosed with Peripheral neuropathy.

\section{Data collection procedure:-}

After taking clinical history, 80 patients were included in the study with clinical features of renal failure; all patients were on hemodialysis, in department of dialysis, at Al-Jabar Nephrology center in Al-Ahasa. Informed consent was taken from each patient.

Detailed history was obtained. Frequency of neuropathy was detected by clinical examination of the sensations (touch, pain, position, vibration) supervised by consultant. Then according to data, we categorized patients according to age, gender, HTN, DM, the presence of peripheral neuropathy and the affected sensations. Data was entered using excel. Frequency and percentage of neuropathy was calculated with proportions of presence or absence of involvement of clinical symptoms.

\section{Discussion:-}

Neuropathy is the late complication occurring in the uremic nephropathy. It has also been resulted from nutritional deficiency, chronic kidney disease and diabetic nephropathy. It's outcomes generally not tolerated, and its main effect regarding sensations include pain, paraesthesias, amputations and disabilities (vagner, 2011). Hot feet sensation a less complications reported in $42 \%$ of cases. However, neuropathy is associated with Autonomic dysfunction. In patients with ESRD, sensory ataxia is an important complication. Physiotherapy is the main stay to prevent this ataxia. However, physiotherapy contribute to maintain independence as possible and insure easier mobility. Sensory neuropathy may cause an increased susceptibility to foot ulcer including infections, gangrene, amputations. The literature reveals many reported instances of amputations associated with uremic neuropathy, as well as report of amputations in CKD (PAUL, 1999). The national institute of neurological disorders (NINDS) recommends adapting self-care exercise. In some other studies, more complex treatments recommended with systemic diseases. Although our patients had renal failure, given the irreversible nature of disease with dialysis, uremia toxicity prevention recommended by American family physician (AFP) is kidney transplant. Reported that patients with renal failure who were complicated with neuropathy, had increase chance of morbidity. The median overall morbidity rate was $13 \%$ (hari, 2013). Our patients had significant loss of sensations clinically but further evaluation by nerve conduction studies, could not be performed. However, under clinical findings, neuropathy was confirmed by examination. In study we focused on peripheral neuropathy, but there are other studies (Doreen, 2011) on primarily axonal sensorimotor polyneuropathy and they noted that peripheral neuropathy occurs in 50-60\% of patients who require hemodialysis. In our patients, we use clinical examination to detect peripheral neuropathy , and we found 50 of 80 patients were having peripheral neuropathy $(62.5 \%)$, of which 39 were diabetics (78\%) and 47 were hypertensive (94\%), and we found similar results in other studies (Aggarwal, 2013)

\section{Result:-}

Peripheral neuropathy was diagnosed using the previously mentioned methods and the results came as the following:

\begin{tabular}{|l|l|l|}
\hline Details & Number of all patients & Affected \\
\hline Number of Male & 38 & 21 \\
\cline { 2 - 3 } & $48 \%$ & $42 \%$ \\
\hline \multirow{2}{*}{ Number of Female } & 42 & 29 \\
\cline { 2 - 3 } & $53 \%$ & $58 \%$ \\
\hline Total & $\mathbf{8 0}$ & $\mathbf{5 0}$ \\
\cline { 2 - 3 } & & $\mathbf{6 2 . 5 0 \%}$ \\
\hline Details & Number of patients & \\
\end{tabular}




\begin{tabular}{|l|l|}
\hline Number of all patients have DM (out of 80) & 47 \\
\cline { 2 - 2 } & $58.75 \%$ \\
\hline Number of affected patients have DM (out of 50) & 39 \\
\cline { 2 - 2 } & $78 \%$ \\
\hline Number of all patients have HTN (out of 80) & 72 \\
\cline { 2 - 2 } & $90 \%$ \\
\hline $\begin{array}{l}\text { Number of affected patients have HTN (out of } \\
50)\end{array}$ & 47 \\
\cline { 2 - 2 } & $94 \%$ \\
\hline
\end{tabular}

\begin{tabular}{|l|l|}
\hline Type of lost sensation & Number of patients \\
\hline vibration & 19 \\
\hline position & 20 \\
\hline pain & 6 \\
\hline Touch & 22 \\
\hline All & 11 \\
\hline
\end{tabular}

Out of 80 patients of renal failure, which were on hemodialysis, 50 patients were having peripheral neuropathy $(62.5 \%)$, of which 39 were diabetics $(78 \%)$ and 47 were hypertensive $(94 \%)$. Out of 42 female patients, 29 were affected (58\%) and out of 38 male patients 21 were affected (42\%). Out of the whole 50 affected patients, the fine touch sensation was affected in 22 of them (44\%), position sensation was affected in 20 of them (40\%), and vibration was affected in 19 of them (38\%), while pain was affected in only 6 of them (12\%). We found total impairment of all sensations in 11 of them (22\%) and all of these were diabetics for more than 10 years.

This reflects the affected tracts of sensory pathway, as the fine touch and proprioceptive sensation and vibration are the most affected sensations, this indicates that the neuropathy has more impact on the dorsal Column-Medial Leminiscal Pathway, and the least affect is on the lateral spinothalamic tract; which carries the pain sensation.

We also found that the duration of hemodialysis doesn't correlate with the progression of neuropathy, as many patients were affected in all of examined sensations while they just started hemodialysis, but it is correlated with other factors such as the control of the other co-morbidity in which here is diabetes, and the intensity of the hemodialysis and the progression of the underlying pathology of the kidneys.

\section{Conclusion:-}

Patients with end stage renal disease in Al-Ahsa have high frequency of peripheral neuropathy, which is more observed in patients with underlying diabetes. Control of diabetes and improved uremic control by frequent hemodialysis improve uremic neuropathy.

\section{Limitations:-}

-patients' education level sometimes affects the understanding and the perception of the clinical examination as the examination is subjective to what they feel or think.

- Patients' psychological status at time of examination affects the result either by exaggeration because of depression or denying because of fear.

-couldn't asses the nerve conduction velocity studies and get more accurate results because of lacking of facilities and access to the NCVS.

-no assessment of the crude touch and temperature sensations.

\section{References:-}

1. Hari K. Aggarwal, Sushma Sood, Deepak Jain, Vipin Kaverappa \& Sachin Yadav (2013). Evaluation of spectrum of peripheral neuropathy in predialysis patients with chronic kidney disease.1323-1329

2. Doreen. Doreen T. Ho,Nancy M. Rodig, Heung B. Kim, Hart G. W. Lidov, Frederic D. Shapiro, G. Praveen Raju, Peter B. Kang (2011). Rapid Reversal of Uremic Neuropathy Following Renal Transplantation in an Adolescent.1399-3046.

3. PAUL. (1999). Nontraumatic lower extremity amputations in the Medicare. vagner, b. (2011). uremic neuropathy a review.

4. Ria Arnold, Bruce A. Pussell, Timothy J. Pianta, Virginija Grinius, Cindy S-Y. Lin, Matthew C. Kiernan, James Howells, Meg J. Jardine, Arun V. Krishnan 2013 Effects of Hemodiafiltration and High Flux Hemodialysis on Nerve Excitability in End-Stage Kidney Disease 10.1371/journal.pone.0059055. 